\title{
El genitivo-ablativo singular del indoeuropeo arcaico: viejas y nuevas continuaciones célticas
}

\author{
Patrizia de Bernardo Stempel \\ Universidad del País Vasco \\ patrizia.debernardo@ehu.es
}

Recibido: 30 de agosto de 2010

Aceptado: 30 de noviembre de 2010

\section{RESUMEN}

Revisión de lo que se ha dicho hasta la fecha en pro y en contra de los supuestos genitivos ablativales de los temas en -o- celtibéricos y lepónticos, indicando además cuántas otras formas del celta continental - tanto primitivo como propiamente galo- y goidélico podrían ser explicadas de la misma manera, es decir como continuaciones del genitivo-ablativo singular no diferenciado del indoeuropeo arcaico. Además del Calendario de Coligny y de la plaquita de Chamalières, se tratan las leyendas monetales RIG-IV-84f., 91, 277 así como las inscripciones RIG-G-24 y 250s. y, en particular, G-154 de Villelaure y *L-131 de Vindonissa/Windisch.

1. Introducción; 2. Los genitivos temáticos en -o y los supuestos ablativos en -uz del celtibérico; 3. Las formas en $-u$ del corpus céltico cisalpino y su interpretación; 4. El genitivo-ablativo singular en indoeuropeo; 5 . Viejas y nuevas continuaciones célticas del genitivo-ablativo singular temático protoindoeuropeo.

Palabras clave: indoeuropeo, celta, celtibérico, celtoligur, lepóntico, galo, goidélico, ogam, irlandés, declinación nominal, fonética histórica, leyendas monetales, Chamalières, Coligny, Villelaure, Vindonissa, Windisch.

\begin{abstract}
State of the art of what has been said in favour of and against the alleged ablatival genitives of the Celtiberian and Lepontic $o$-stems. The discussion extends to further Celtic forms - Goidelic as well as Gaulish and also Primitive Continental — which could be explained along the same lines, i.e. als continuing the arcaic Indo-European undifferentiated genitive-ablative singular, such as the coin legends $R I G$-IV-84f., 91, 277 or the inscriptions RIG-G-24 and 250f. and, in particular, G-154 from Villelaure and *L-131 from Vindonissal Windisch; the Calendar of Coligny and the Tablet of Chamalières are also discussed.

1. Introduction; 2. Thematic $o$-genitives and alleged $u z$-ablatives in Celtiberian; 3. $u$-endings in the Cisalpine Celtic corpus and their interpretation; 4. The Indo-European genitive-ablative singular; 5. Old and new Celtic instances of the Proto-Indo-European thematic genitive-ablative singular.
\end{abstract}

Key words: Indo-European, Celtic, Celtiberian, Ligurian Celtic, Lepontic, Gaulish, Goidelic, ogam, Irish, noun declension, historical phonetics, coin legends, Chamalières, Coligny, Villelaure, Vindonissa, Windisch. 


\section{INTRODUCCIÓN}

La presente contribución ${ }^{1}$ se propone revisar lo que se ha dicho hasta la fecha en pro y en contra de los supuestos genitivos ablativales de los temas en -o- celtibéricos y lepónticos, indicando además qué más formas del celta continental - tanto primitivo como propiamente galo - podrían ser explicadas de la misma manera.

La historia de la cuestión empieza en 1977, cuando K.H. Schmidt interpreta el genitivo singular en - $O$ de los temas en -o- celtibéricos como un resto del ablativo temático indoeuropeo en *-ōd (cf., además, $i d$. 1995). Más tarde, J. de Hoz mostró en 1990 cómo aquellas formas de nombres personales acabadas en $-u \mathrm{o}-i o^{2}$, que - dentro del corpus que se suele llamar lepóntico- se hallan en la posición normalmente ocupada por un genitivo ${ }^{3}$, podían estar relacionadas con las formas celtibéricas de genitivo en - $O$. A continuación, yo misma en 1991 y 1992/93 expliqué — seguida por Ködderitzsch (1992/93) — unos fósiles léxicos irlandeses como restos del mismo tipo de ablativo. Finalmente, en 2001/03, llegué a la conclusión de que el ablativo singular que considerábamos que subyace a todas estas formas genitivales no fue un ablativo discreto, separado del genitivo singular, sino que tuvo que ser más bien la primera forma de la cual dispusieron los temas en -o- a la hora de expresar una relación de procedencia, pertenencia y separación $n^{4}$.

\section{LOS GENITIVOS TEMÁTICOS EN -O Y LOS SUPUESTOS ABLATIVOS EN -UZ DEL CELTIBÉRICO}

2.1. Aquellos lingüistas ${ }^{5}$ que aceptaron — acríticamente - el vuelco dado a la gramática celtibérica en 1995 tuvieron que rechazar la hipótesis de un genitivo ablatival, dado que la nueva moda o «new look» ${ }^{6}$ pretendía que todas las terminaciones indoeuropeas en *-ôd hubiesen dado -uz en celtibérico; la existencia de dos diferentes continuaciones celtibéricas de un mismo morfema de ablativo era en consecuencia insostenible para ellos.

En particular, se dejó de considerar sustantivos celtibéricos en -uz del tipo de las leyendas monetales Ka.r.a.l.u.z y U.s.a.m.u.z como formas fonéticamente modernizadas de los nominativos plurales temáticos *Ka.ra.l.o.s y *U.s.a.m.o.s que indicaran [Gral:o:s] 'Los vecinos de Grallum' y, respectivamente, [Usamo:s] 'Los vecinos de Uxama'7. En

\footnotetext{
${ }^{1}$ Quiero expresar a Eugenio Luján mi agradecimiento por su amable revisión de mi castellano. Este trabajo desarrolla algunas cuestiones que de forma más resumida aparecen en Luján y García Alonso (eds., 2010).

${ }^{2}$ Antes de llegar a -iu, es decir *-yō\#.

${ }^{3}$ Sea este de procedencia — como el genitivo del nombre paterno o de un patronímico- o de posesión.

${ }^{4}$ La contribución fue muy bien recibida por W.P. Lehmann y B. Bauer; cf. también las reseñas de Meid (2002: 23) y Gusmani (2005).

${ }^{5}$ Para una discusión más serena de los materiales en cuestión - y siguiendo un uso que dio buenos resultados en otra ocasión (Celtes et Gaulois I: 33-56) - , no mencionaré en mi texto el nombre de aquellos estudiosos cuyas ideas no puedo compartir, limitándome a discutir simplemente sus posturas; sus obras aparecen, por supuesto, citadas en la bibliografía al final del presente artículo.

${ }^{6}$ Parece que el prestigio académico de su iniciador — y quizá también su entorno hispánico— haya(n) sido suficiente(s) para convencer de la bondad de una teoria tan poco económica a aquellos estudiosos internacionales que hasta ese momento no se habían ocupado de paleohispanística.

${ }^{7}$ La preservación de la desinencia originaria se aprecia en S.e.ki.s.a.m.o.s [Segisamo:s] 'Los vecinos
} 
su lugar, dichas leyendas pasaron a interpretarse como formas de ablativo singular en *-o $d$, sin tener en cuenta que el ablativo es en general muy raro en monedas ${ }^{8}$, para no hablar de aquellas lepónticas y galas, donde tan sólo aparecen nominativos y genitivos 9 .

En contra de esta teoría habla aún más la discrepancia de género que hay a veces entre los sustantivos atestiguados en las leyendas y los topónimos relacionados, como en el caso de U.s.a.m.u.z vs. U.s.a.m.a. Dado que U.s.a.m.u.z no puede ser el ablativo de un tema en $-\bar{a}$ como el segundo, es decir el ablativo del nombre de una de las dos ciudades que se conocen como Uxama, los responsables del «new look» tuvieron que suponer que lo fuese de **Uxamos o de **Uxamon, o sea del nombre de una tercera población - sin embargo no documentada históricamente.

Mientras tanto, sin embargo, se ha podido demostrar que la terminación -uz tras una consonante sonora es el resultado de la sonorización de la sibilante sorda $*_{-S}$ indoeuropea y celtibérica que ha tenido lugar en sílabas átonas que empiezan por fonemas sonoros ${ }^{10}$; esto significa que la secuencia -R $u z$ que aparece p.ej. en Ka.r.a.l.u.z, U.s.a.m.u.z se remonta simplemente a la terminación *-Rus que continuaba el *-Rōs celtibérico arcaico, celta antiguo e indoeuropeo de sus nominativos de plural.

La misma regla explica por qué la desinencia de las leyendas monetales arcaicas en -kos mencionadas arriba en la $\mathrm{n} .7$ nunca se sonorizó en **-kVz .

Asimismo, la regla permite dar cuenta de las leyendas monetales Ba.r.s.Ku.n.e.z / Ba.s.Ku.n.e.z — que problemente representan *Braskunez - como originarios nominativos de plural *Brask-un-es del tema en - $n$ que indica un grupo de población, con la sonorización regular detrás de una sonante; no obstante, la nueva moda pretende que se trate del ablativo singular de un topónimo con tema en nasal y por lo tanto atípico ${ }^{11}$; análogamente, la leyenda S.e.ko.bi.r.i.ke.z no tiene por qué ser un ablativo singular toponímico, pudiendo representar el nominativo plural *Segobrig-es con sonorización regular de la sibilante final detrás de una oclusiva sonora (la forma en velar podía designar a los habitantes habiendo el singular asumido apariencia de tema en -i-: Segobri-s).

La misma sonorización puede dar cuenta de los alomorfos -as/-az de la desinencia de genitivo singular de los temas en $-\bar{a}$, como en N.o.u.a.n.tu.ta.s vs. A.ka.i.n.a.z : aunque el «new look» los haya explicado como ablativos de singular, su connotación semántica es más probablemente — con Isaac (2002: 4s) y por otras varias razones ${ }^{12}$ -

de Segisama', cuyo arcaísmo se percibe, además, de en la sibilante intervocálica -s- todavía sin sonorizar; igualmente, en las varias leyendas monetales celtibéricas en -os que expresan nombres de habitantes derivados por medio de los sufijos velares -ako- o -iko-: E.ku.a.l.a.Ko.s, A.r.Ka.i.l.i.Ko.s, Ka.1.a.Ko.r.i.Ko.s etc.; cf. DBSt (2004/05: 557s.).

${ }^{8}$ Se notará que los pocos ejemplos citados por el colega iniciador de la nueva moda proceden de monedas con leyendas en dialectos itálicos, acuñadas por ciudades de la Italia del Sur; sin embargo, fue la amonedación griega — donde no se hallan ablativos o dativos- la que inspiró las primeras acuñaciones célticas, tanto celtibéricas como de otras áreas. Más argumentos en contra alega Meid (2000: 20s.).

${ }^{9}$ Cf. LKA s.vv. «Gallogriechische —», «Gallolateinische Inschriften» y «Lepontisch»; CIT 2: pp. 493-514; DBSt (1999/2001: 328).

${ }^{10}$ DBSt $(2004 / 05)$.

${ }^{11}$ Cuatro argumentos en contra enumera DBSt (1998/2007A: 149 con la n. 29). Acerca de ésta y de la siguiente leyenda cf. también ead. (2004/05: 558).

${ }^{12}$ Como el hecho de que, de otra manera, el magistrado llamado pi.n.ti.s, en vez de regir siempre el 
el resultado de la indiferente función de genitivo-ablativo que el morfema -ās desempeñó originariamente en el protoindoeuropeo ${ }^{13}$. De esta manera, es también más fácil dar cuenta de la desinencia - $a$ que continúa el nuevo y específico ablativo en *-ād de los temas en $-\bar{a}$, producto de la analogía con los temas en $-o^{14}$.

Tampoco las formas verbales celtibéricas en $-u z$ como ta.tu.z, es decir [datuz] 'tiene que dar-él' se pueden — entre otros ante las nueva atestiguaciones celtibéricas de imperativo en -to y $-t u^{15}$ - retrotraer a la simple desinencia *-tôd de imperativo 'futuro'; esas desinencias son, más bien, el producto de la fusión de dicho *-(t)ōd con una $-s$ pronominal, de la misma manera que las formas verbales celtibéricas en $-e z$ surgen de la amalgama de ${ }^{*}-e t+-s$ : en ambos casos, la dental de una desinencia de $3^{\mathrm{a}}$ persona singular se fundió con la sibilante del pronombre sujeto que le correspondía ${ }^{16}$.

Incluso desde una mirada más general, no se puede considerar el «new look» una alternativa satisfactoria a la visión tradicional de la gramática celtibérica, ni con respecto a la fonética (a), a la morfosintaxis (b) o a la metodología (c).

En cuanto a la primera (a), se dirá que no hay razón para pensar que una simple * $d$ en posición final haya sido en primer lugar preservada y luego transformada en $* *-z^{17}$, cuando una $*-d$ - originaria se encuentra preservada en celtibérico incluso en posición intervocálica al interior de una palabra, mientras que las fuentes de $-z$ - son más bien el nexo *- $d y$-, los grupos de dental+dental/sibilante y también las $-s$ - en entornos sonoros.

En cuanto a la morfosintaxis (b), se resaltará —además de los problemas que conciernen a los varios ablativos ${ }^{18}$ - que (1) aunque en un principio «nominativo o genitivo difícilmente dejarían de estar atestiguados» ${ }^{19}$, el 'nuevo' corpus celtibérico no contendría ni un solo ejemplo de nominativo plural temático ${ }^{20} ;(2)$ un nominativo plural celtibérico en *-oi como el que se postula no encajaría bien en el contexto cronológico de los nominativos plurales célticos de temas en -o-; (3) la ratio - perfectamente lógica desde una mirada numismática — que subyace a la distribución complementaria de las leyendas monetales en -kos (halladas en el noroeste del territorio celtibérico y correspondientes a nominativos plurales arcaicos como los de las leyendas galas del norte ${ }^{21}$ ) y aquellas en -kom (halladas en el este y en el sur del territorio celtibérico, a menudo acuñadas por cecas antiguas y correspondientes a genitivos plurales arcaicos

mismo caso - genitivo - en Botorrita I.B, tendría que regir una vez el genitivo y tres veces el ablativo; más detalles en DBSt (2008/10: 134s.).

${ }^{13}$ Véase abajo $§ 4.1$.

${ }^{14}$ Acerca de cib. s.u.a y M.e.ta.m.a — que de otra manera se quedarían sin explicar- y de unas continuaciones galas del mismo morfema, cf. DBSt (1992/93: 40s.), (1998/2007a: 160) y (2000a: 187 n.20).

${ }^{15}$ Se trata de cib. esto (Arenas et alii, 2001) y datu (De Bernardo et alii, 2010).

${ }^{16}$ Cf. ahora — siguiendo a Eska (1989) y en la estela de Fleuriot (1981: 91) y De Hoz (1983/86: 57) DBSt (2009: 692).

${ }^{17}$ No menos de seis razones para rechazar esta hipótesis expuso entre otros DBSt (1999/2001: 182s.); cf. también ead. (2004/2005), que continúa y en parte modifica mi estudio de 1998/2007 (в).

${ }^{18}$ Más detalles y objeciones entre otros en mis estudios de 1999/2001 (pp. 322-323), 1998/2007A (pp. 161-164), 2001/03 (p. 41s.) y $1991^{\text {a }}$ (p. 210).

${ }^{19}$ De Hoz (2005: 421).

${ }^{20}$ Dado que s.to.te.r.o.i en el $4^{\circ}$ bronce de Botorrita es un pronombre y o.bo.i a.l.a.bo.i una correlación disyuntiva (DBSt, 2002: 98 n. $41 \mathrm{y}$, respective, 2004).

${ }^{21}$ Cf. el mapa en Burillo (20082: 167) y Arenas et alii (2001: 215s.). 
como los de las leyendas galas del sur, por lo tanto afines a las leyendas griegas) cedería el paso a una alternancia inmotivada entre nombres de acuñaciones masculinos - sin paralelos - (supuestos nom.sing. en -kos) y otros nombres de acuñaciones neutros - también sin paralelos- (supuestos nom.sing. en -kom). A dicha suposición se opone, además, la existencia, dentro del corpus hispanocéltico de inscripciones romanas, de numerosos genitivos plurales en -om y -on a partir de nombres de agrupación familiar ${ }^{22}$; (4) los supuestos ejemplos de un caso instrumental en $-u$ son más bien formas abreviadas de genitivo plural, como p.ej. la leyenda monetal Ko.l.o.u.n.i.o.ku, exactamente correspondiente a la leyenda $C_{L O V N I O Q}(U M)$ en alfabeto latino y que representa $K^{o}$ louniok ${ }^{u}($.$) , con -o- y - u\# como vocales mudas; (5) la nueva teoría dejaba sin$ explicar el genitivo temático singular celtibérico en -o.

Finalmente, aunque no por último, la nueva teoría supuso un gran paso atrás con respecto a la metodología (c), siendo impulsada por el deseo de eliminar cualquier tipo de variación diacrónica, diatópica y diastrática durante todo el periodo de duración de la escritura celtibérica y dentro de todo el corpus celtibérico ${ }^{23}$.

No hay, por lo tanto, razones para excluir que el morfema original temático ie. *-ōd continuara regularmente como $-\bar{o}$ en celtibérico arcaico, lo que, sin embargo, hubiera tenido que llegar a **-u en celtibérico clásico.

2.2. Los datos que acabamos de discutir indican con claridad que la nueva cronología relativa que, con respecto al desarrollo de la $* \bar{o}$ indoeuropea, considera tan sólo el paso *-ō- $>-\bar{a}$ - como perteneciente al estadio del celta común ${ }^{24}$ no se basa con exclusividad en la continuación del morfema ${ }^{*}-\bar{o} d$ del genitivo ablatival —a diferencia de cuanto haya podido escribir algún crítico mal informado.

Con respecto a la secuencia ie. ${ }^{*}-\bar{o}(\mathrm{R} / \mathrm{C}) \#$ en posición final, y teniendo en cuenta todos los datos del celta antiguo - y no sólo los que proceden de las Penínsulas Ibérica y Apenínica一, se pueden diferenciar los siguientes estadios ${ }^{25}$ :

\begin{tabular}{|c|c|}
\hline & $\bar{o}$ en posición final absoluta o delante de una voca \\
\hline
\end{tabular}

\begin{tabular}{|l|lll|}
\hline II. & $\bar{o}$ en posición final delante de una consonante \\
\hline & $*_{-} \bar{o}+\{\mathrm{d} / \mathrm{ns} / \mathrm{s} / \mathrm{m} / \mathrm{r}\}$ & $\begin{array}{l}\text { se quedaron primero como } \\
\text { y luego evolucionaron a }\end{array}$ & $\begin{array}{l}-\bar{o} /-\bar{o} s /-\bar{o} m /-\bar{o} r \\
-u /-u s /-u m /-u r\end{array}$ \\
\hline
\end{tabular}

Dicha cronología da cuenta, además de los nominativos plurales en -os y de los genitivos plurales en -om del celtibérico arcaico, del imperativo celtibérico arcaico esto $<*$ estōd, de los genitivos plurales hispanocélticos en -om y -on y de unas formas

\footnotetext{
${ }^{22}$ Cf. la discusión de González Rodríguez (1986) en DBSt (1991A: 210s.).

${ }^{23}$ Cf. DBSt (2004/05: 540-541).

${ }^{24}$ DBSt (1991A y 1992/93).

${ }^{25}$ DBSt (1992/93: 40).
} 
residuales galas como el acusativo pronominal plural $\operatorname{sos}^{26}$, de la $\bar{o}$ que todavía se preserva en unas formas lepónticas como Esopnio (cuya -yo\# se opone a la terminación $-y u$ ya alcanzada por los nominativos de los temas en $-n$ - y al $-y u i$ de los dativos de de temas en - $O$ - y parece, por lo tanto, retrotraerse más bien a $\left.*-y \bar{o}(d)^{27}\right)$ y quizás el ablativo Mešiolano (CIT 140 y p. 480).

Pero el hecho de que la desinencia del singular del genitivo temático celtibérico aparezca siempre como -o -incluso en los textos más tardíos, cf. Ta.u.r.o 'de Tauros', a.r.e.s.ta.l.o 'del arestalos o presidente' en Botorrita 1- ha sido utilizado por algunos estudiosos como razón para descartar su origen ablatival. Sin embargo, la cronología relativa con tres estadios vocálicos que acabamos de ilustrar implica que durante el segundo estadio el sistema celtibérico de desinencias singulares fuera del tipo A en la declinación temática:

\begin{tabular}{|lll|l|}
\hline \multicolumn{2}{|c|}{ A } & \multicolumn{1}{c|}{ B } \\
\hline Nom. & $-o s$ & & $-o s$ \\
Gen. & $-\bar{o}$ & $\left(\right.$ si del ie. $\left.{ }^{*}-\bar{o} d\right)$ & $-o$ \\
Dat. & $-u i$ & $\left(<\mathrm{IE} *_{-} \bar{i}\right)$ & $-u i$ \\
Ac. & $-o m$ & & $-o m$ \\
Loc. & $-e i$ & & $-e i$ \\
\hline
\end{tabular}

Es evidente que un sistema de tipo A podía con facilidad llegar, por analogía, a un sistema de tipo $\mathrm{B}^{28}$, siendo en $\mathrm{A}$ el morfema * $\bar{o}$ hipercaracterizado tanto con respecto a la desinencia -os como a la desinencia -om, de las cuales - pese a tener la misma coloración vocálica - dos rasgos lo separaban: [+Vowel-Length] y [-Final Consonant].

De hecho, si tenemos en cuenta que un sistema del tipo B es exactamente lo que tenemos en celtibérico, podemos identificar el estadio A con un nivel arcaico de la misma lengua:

\section{$\mathrm{B}:$ Celtibérico $($ clásico $)=\mathrm{A}:$ Celtibérico arcaico}

2.3. Mucho menos económicas y plausibles han sido las otras soluciones barajadas para dar cuenta de la -o breve del morfema genitival celtibérico, tanto que el «new look» acabó negando la existencia de una explicación histórico-comparativa satisfactoria.

Muy a menudo se postula la extracción de un morfema de genitivo a partir de aquellos sintagmas donde se supone que un nombre común sigue a un pronombre demostrativo. En particular, se ha supuesto que una secuencia de genitivo plural del tipo de *soisōm wirōm 'de estos hombres' hubiera dado — a través de ${ }^{* *}$ soiso wiroiso y puede que influido

\footnotetext{
${ }^{26}$ Sin embargo, el -šo- infijado de la bilingüe de Vercelli aparece ahora ser el acusativo singular neutro del pronombre demostrativo n o c a t a f ó r i c o *sto- (cf. DBSt, 2008/e.p.).

${ }^{27}$ No obstante, un posible ejemplo de -iu lepóntico genitival $(<-i o)$ podría atestiguarse en CIT 269.

${ }^{28}$ DBSt (1991A: 217) y (1992/93: 50s.) discute otras hipótesis analógicas anteriores junto con objecciones anti-analógicas. Se advertirá, al margen, que la reconstrucción operada por Shields (2005: 238) de una *-o genitival a partir de ie. *-o-+-o requeriría el mismo tipo de abreviación analógica postulada arriba, dado que en celta también las *-o primarias en final de palabra llegaron a $-u$.
} 
también por **soisās bnās - un singular **soiso wiro, aunque la forma del genitivo singular del pronombre es muy hipotética. Pero, ¿cuál fue antes el morfema de genitivo singular temático en celtibérico? ¿Y por qué se necesitó un cambio? ¿Porqué la reestructuración se hizo a partir del plural? Y ¿por qué no se mantuvo el mismo*soiso wiroiso, si es que en algún momento de verdad hubo en celtibérico un genitivo temático de tipo -osyo? ¿No sería extraño que se hubiera introducido un morfema de este tipo para luego eliminarlo? Y finalmente, aun aceptando que la nueva terminación -o fuese debida a economía, ¿por qué no habría ninguna otra evidencia de Gruppenflexion en los demás casos ${ }^{29}$ ?

Podemos, por lo tanto, concluir que la hipótesis de un genitivo ablatival en celtibérico no es formalmente implausible.

\section{LAS FORMAS EN -U DEL CORPUS CÉLTICO CISALPINO Y SU INTERPRETACIÓN}

3.1. Como he adelantado en $\S 1$, en 1990 J. de Hoz aprovechó la oportunidad, lingüísticamente interesante, de establecer una conexión diacrónica entre el genitivo singular temático celtibérico y los morfemas $-u$ /-io que aparecen en posición genitival en el corpus lepóntico.

Antes de pasar a los detalles de dicha explicación, queremos ante todo recordar que (1) un corpus cualquiera nunca contiene o refleja tan sólo una lengua/ dialecto/ variedad lingüística ${ }^{30}$. En particular, algunos de los elementos que integran el corpus que llamamos lepóntico se describirían mejor - con Rubat Borel (2009) - como pertenecientes a la cultura de Golasecca, otros como galos; (2) si bien sabemos ahora que la glotogénesis céltica se desarrolló de manera gradual y sin separaciones repentinas y radicales, no todas las isoglosas lepónticas tienen por qué ser arcaísmos: hay incluso unas cuantas innovaciones lepónticas que separan este dialecto del galo común ${ }^{31}$.

Los argumentos específicamente lepónticos en favor de una interpretación genitival temática de las formas en - $u$ /-io fueron básicamente de tres tipos, semánticos (I), morfológicos (II) y estructurales (III) ${ }^{32}$.

La ventaja semántica (I) es la mejor comprensión de fórmulas onomásticas como:

Aškonetio Pianu 'de Bivonos, del hijo de Adgonnetos', que corresponde al tipo arcaico $\{$ Patroním. $+\mathrm{NP}\}$, es decir a una secuencia de Determinans + Determinatum ${ }^{33}$;

Namu Esopnio 'de Namos, del hijo de Sinmiedo' y Oletu Amašilu 'de Olletos, del hijo de Ambatios', pertenecientes al tipo \{NP + Patroním. $\}$;

${ }^{29}$ También el sintagma cib. ${ }^{* *}$ s-oso Karik-oso — postulado por otro estudioso y para el cual tampoco hay apoyos - se hubiera quedado igual, dado, además, que un supuesto reanálisis hubiera proporcionado **sos-o Karikos-o. Y el no menos hipotético ** sosyo wir $\overline{~ d e ~ o t r o ~ l i n g u ̈ i s t a ~ h u b i e r a ~ t e n i d o ~ q u e ~ p r o p o r c i o n a r ~ p r i m e r o ~}$ **sosyo wiryo. Más detalles acerca de este tipo de reconstrucciones en DBSt (1998/2007A: 164) y (2001/03: 42).

${ }^{30}$ Cf. DBSt (2005/06: 35s. y 43).

${ }^{31}$ DBSt (2005/06: 41), revisado en ead. (2008/e.p.: § I).

${ }^{32}$ En estos párrafos, los materiales aportados por De Hoz (1990: 319ss.) se citan ya con varios añadidos a cargo de la autora del presente artículo.

${ }^{33}$ DBSt (1992/93: 50 n. 44); otros ejemplos de este mismo tipo de secuencia en fórmulas onomásticas célticas ofrece Motta (2002/09: 305 n. 41). 
Uvamokozis Pliale $\theta u$ 'Uvamogostis, (hijo) de Blialetos', del tipo \{NP + Gen.padre\}. El tipo \{Gen.padre + NP\} parece ser representado por Kiketu Retalos '(hijo) de Cingetos Rodallos ${ }^{34}$, dado que una interpretación como ${ }^{* *}$ 'Cingeton, hijo de Retos', o sea del tipo $\{\mathrm{NP}+$ Patroním. $\}$ se tendría que descartar por razones estructurales ${ }^{35}$.

Una secuencia de dos genitivos como en los dos primeros tipos citados podría dar cuenta también de Minuku Komoneos como 'de Minucos Comonis', aunque no podemos decir si el nominativo de tema en $-i-$ *Komonis es un patronímico u otro tipo de atributo ${ }^{36}$.

Se advertirá que la fórmula **Letiu (or Uetiu) Sivilios, citada por algunos autores, procede de una inscripción falsa ${ }^{37}$.

En el plano morfológico (II), si se tratara de temas en - $O$ - y no de temas en - $n$ - como tradicionalmente se ha asumido, se eliminaría (a) la desproporción entre los pocos genitivos, dativos u otros casos de temas lepónticos en nasal y la gran cantidad de supuestos nominativos de singular y también (b) aquella entre la frecuencia de temas en nasal en el lepóntico reconstruido y en los demás dialectos y lenguas.

Además (IIIa), si se tratara de temas en nasal, la proporción de nombres hipocorísticos (como el nominativo Deu, $<\mathrm{Teu}>$, que, actuando como sujeto en Vergiate, representa la forma abreviada * $D \bar{e} w \bar{o}(n)$ de un idiónimo teofórico, del tipo de *Deiwogenos 'Nacido de Dios' o quizás *Deiworis ${ }^{38}$ ) hubiera sido estadísticamente demasiado elevada.

Finalmente, siempre desde la perspectiva estructural (IIIb), los nombres personales involucrados a menudo apuntan a que han sido temas en - $O$ - (y no en $-n-$ ), y en particular unas cuantas formas lepónticas en - $u$ /-io (A) por tener correspondientes onomásticos que son temas en $-O-\mathrm{u}-\bar{a}$, como en el caso de ${ }^{39}$

- la forma Atepu, para la cual se conocen Atepa y el más arcaico Atekua $/ A T E C V A^{40}$,

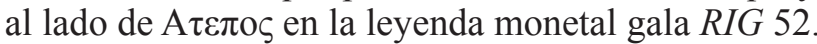

Además,

— compárese la forma Pianu con BIVONIA y BIANIUS desde Brescia y, respectívamente, la Liguria;

- la forma Bou — con la misma caída de la - w- que acabamos de ver en $<$ Pianu $>$, ['Bianu], desde * $G^{w}$ íwonōd y en $<$ Teu $>$, ['De:u], desde *Dēwō $(n)$ — compárese con el segundo elemento del compuesto temático $<$ Toutiopovos $>$ que aparece sobre monedas, y también con BoVIUS / BOVIA;

— compárese la forma Kiketu con CINGETIUS / CINGESSUS;

${ }^{34}$ El idiónimo principal podría continuar un originario *Ro-dallos 'Realmente ciego', si bien Prosdocimi (2002/09: 89) lo interpreta como un derivado de *recto-.

${ }^{35}$ Véase ad IIIb A y B.

${ }^{36}$ Véase también la fórmula onomástica genitival Komoneos Vađsileos procedente de la misma localidad (Stabio $/ \mathrm{CH}$ ); la desinencia - eos de los temas en - $i$ - ha sido explicada como contaminación del morfema arcaico *-y-os de genitivo-ablativo singular en la declinación involucrada y su alomorfo *-ei-s, contaminación resultante en $*_{-} e^{i}$ os (cf. DBSt, 2001/03: 38). En cuanto al Komonos que se atestigua en las cercanías de Verona (CIT 251) sobre un fragmento cerámico, podría representar tanto el genitivo de un más antiguo tema en nasal — como el que todavía se aprecia en el sello de sigillata Cомо (NTS-Index 104) - o el nominativo de una variante tematizada.

${ }^{37}$ De Cernusco sul Naviglio, cf. CIT 137* (vol. II: 613) con bibliografía.

${ }^{38}$ Acerca de los nombres teofóricos célticos véanse (Meid 2005/08) y DBSt (2007/08).

${ }^{39}$ Para más detalles acerca de los NPP citados a continuación cf. entre otros $C L I$ y NPC.

${ }^{40} \mathrm{La}$ existencia de una capa de 'celta $q$ ' en el corpus lepóntico fue distinguida por DBSt (1990: 30s.). 
— compárese la forma $T a u^{41}$ con DAVOS / DAVUS y con el DAUS 'Flamante' transpadano;

- compárese la forma Tiou con DIVUS y DIVVOGNA ${ }^{42}$;

- compárese la forma Esopnio con $<$ Esopnos $>$ en Garlasco, con los más tardíos EXOBNOS / EXOBNUS / EXSOMNUS, al lado de los cuales se atestigua — con epéntesis- el epíteto teonímico EXOBINOS, además con EXOBNA en Brisino y la más tardía EXOMNA;

- compárese la forma $\mathrm{Kaio}^{43}$ con los itálicos Гóros / GAIUS;

- la forma Minuku con MINUCIA;

— la forma Oletu con OLITIUS ;

- compárese la forma Rešu ${ }^{44}$ con REDSOMAROS y el dálmata RESSIUS;

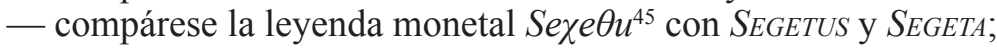

- compárese la forma Uenu con VENNUS / VENNA y VENOCARUS, aunque también se conozca el nombre $V E N N O$;

- compárese la forma Uerkou con VERCUS y con el título de VERGOBRETOS;

- la forma $U i r u^{46}$ con VIROS.

- Tenemos, además, los originarios temas en -o- *Aškonetos (implicado por el genitivo Aškoneti en Stabio, CIT 38) al lado de ADGONETUS / ADGONNETUS, AMBAXIUS con AMASIA y ANTESTUS con ANTESSIUS, a partir de los cuales fueron derivados los nominativos de las formas Aškonetio y, respectivamente, Amašilu y Antešilu, mientras - una vez más - para ninguno de ellos se conoce una variante con tema en nasal.

- Ambiguos son Namu, que se puede comparar tanto con NAMMUS y NAMA como con el $N_{A M}(M)$ o que aparece entre otros como Namuni (dat.) en la Transpadana, y Talu, que puede representar tanto el genitivo de TALUS 'Frente' o de DALLUS 'Ciego' como el nominativo $D_{A L L O}$.

Otras formas (B) apuntan a que han sido temas en - $O--\mathrm{y}$ no en nasal- por estar derivadas por medio de sufijos temáticos, entre ellos

-ilo- en el caso de Amašilu y Antešilu, sufijo que es claramente temático en el caso del *Ritilos implícito en el genitivo Ritili que se halla en la nueva inscripción latènica de Argnou $^{47}$; también se conoce un correspondiente femenino en - $\bar{a}$, cf. -ila en Coimila de Levo. Al margen se advertirá que este sufijo parece haber sido un alomorfo del -alo- que en este territorio se especializó como patronímico ${ }^{48}$.

${ }^{41}$ En Verdello: CIT 286. La traducción de los NPP latinizados sigue NPC: 219.

${ }^{42}$ Acerca de - $v v$-, y en general acerca de los fonemas geminados, véase ahora DBSt (2009/10).

${ }^{43}$ En Arsago: CIT 127; además, el nombre se halla, abreviado, en CIT 254 y en Oderzo como derivado patronímico *Kaialos, implícito en el genitivo Kaialoiso 'del hijo de Gaios'.

${ }^{44}$ Se advertirá que el **Remu inventariado por Lejeune y otros autores era una vieja lectura para este mismo Rě̌u.

${ }^{45}$ Aunque las leyendas monetales lepónticas son en su mayoría nominativos, no podemos excluir a priori

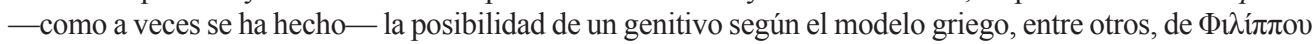

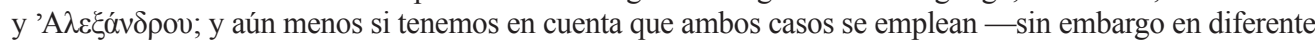
proporción - también en Galia y Britania, cf. DBSt (1991в: 47s.) y LKA s.vv. «Gallogriechische —》 y «Gallolateinische Inschriften». Tampoco hay apoyo alguno para el «[seghetō(n)]» reconstruido por algún autor.

${ }^{46}$ Del cual CIT 258 — si la lectura fuera correcta— podría acaso representar un estadio fonético más arcaico.

${ }^{47}$ Rubat \& Paccolat (2008). Para Atilon- véase a continuación.

${ }^{48}$ Prosdocimi (2002/09: 84ss.) y DBSt (en prep.); acerca del segundo véase ead. (1998: 609s.) con NWÄI: 455s. 
— Cf., además, el morfema -ano- que continua el sufijo *-ono- (NWÄI: 452s.) en posición átona y aparece en la forma $<$ Pianu $>$ que pertenece a un nominativo singular *Bíanos $<*$ Bívanos $<*$ Bíwonos $<$ célt. * $G^{\text {wíwonos }}$ 'El Vivo'. La forma intermedia de nominativo está implícita en los NPP citados arriba en el apartado A, y el anterior tema en nasal *Bivon- está atestiguado en el dativo singular lep. $<$ Pivonei $>49$;

- el sufijo -ato- en $<$ Atlatu $>$, probablemente de $* A ́ t(i) l o s$, que correspondería al A $\tau \imath \lambda \alpha$ de la Narbonense ${ }^{50}$

— el sufijo -eto- (NWÄI: 454s. y 451) en <Kiketu>, del celta *kingetos 'guerrero'; en $<$ Se $\chi \varepsilon \theta 0 v>$, del celta *segetos, o con el sentido del lat. prolis o como «The Holder or Retainer $\rangle^{51}$; en $<$ Oletu $>$, relacionado con el celta * ollo- 'grande, vasto; todo' ${ }^{52}$.

Es verdad que en Bélgica aparece, en el genitivo latinizado $O_{L E D O ́ N I S}{ }^{53}$, un tema en - $n$, fonéticamente modernizado y derivado de este mismo idiónimo, pero sabemos que los pluriderivados o Mehrfachderivative son una categoría lingüística en general comparativamente tardía ${ }^{54}$ : por lo tanto, pese a la existencia del dativo $<$ Atilonei $>$ en Giubiasco en el I siglo a.C., no es metódicamente correcto postularlos para un estadio lingüístico tan arcaico como el lepóntico stricto sensu;

- el sufijo velar -iko- (NWÄI: 327ss.) en la leyenda monetal < Anatikou>, probablemente desde * Andatikos ${ }^{55}$;

- el sufijo velar -uko- (NWÄI: 327ss. y 332) en Minuku, probablemente desde *Mindu-ko-s 'Que lleva una diadema o corona (célt. *mindu ${ }^{56}$ )', con la asimilación $n(n)<* n d$ característica del lepóntico;

— el patronímico -yo- en $<$ Esopnio $>$ a partir de *Exobnios 'hijo de Sinmiedo (célt. *ex-obno-s)' y en $<$ Aškonetio $>$ a partir de *Aškonetios 'hijo del Matador (célt. *Ad-gón(n)-eto-s) ${ }^{57}$.

\subsection{Otros posibles candidatos a genitivos podrían ser}

— $<$ Pokru $>$ en una patera 'Golasecchiana' hallada en Ossuccio (CIT 145), si es que representa un compuesto verbal *bo-gr-o-s a partir de célt. *bow- 'vaca' y el grado cero de la raíz ie. ${ }^{*} g^{\text {'h }} e h_{2} r$ - 'llamar, citar's8;

- Uiku / Uixu en el corpus etrusco y, respectivamente, en un vaso de Sesto Calende (CIT 78), si su nominativo es *wik-ó-s 'combatiente';

— < Uisou $>$ en la estela de Pregassona CIT 279, si está relacionado con VISIUS.

\footnotetext{
${ }^{49}$ Cf. también $<$ Piuonta $>$ en Coccaglio (BS), continuado más tarde por BIONTA, los dativos $<$ Piuotialui $>$ en Davesco y Mezzovico, en los cuales está implícito un patronímico $*<$ Piuotialos $>$ y por consecuencia un idiónimo * Biwontios; además, los ambiguos $<\operatorname{Piuot}()>\mathrm{y}<\operatorname{Piuo}()>$ de Parre y Caravaggio. La propuesta de Tibiletti Bruno (1981: 167) es muy poco convincente.

${ }^{50}$ Un tema en -o-Atilo- está involucrado también en el tema en - $n$ - atestiguado en Giubiasco como dativo Atilonei.

${ }^{51}$ Las dos etimologías en Matasović (2009: 326s.).

${ }^{52}$ Matasović (2009: 136s.), donde se tendría que añadir DBSt (2003).

${ }^{53}$ Con simplificación pretónica de la geminada, cf. la bibliografía en la n. 42 .

${ }^{54}$ NW ̈̈I: 478ss.

${ }^{55}$ Cf. cuanto se dijo en la n. 45.

${ }^{56}$ Matasović (2009: 272).

${ }^{57}$ Más detalles en DBSt (2002/09: 175) y (1998/2007A: 151 con la n. 43).

${ }^{58}$ Para los dos elementos cf. Matasović (2009: 71s. y 153).
} 
- En cuanto a la leyenda monetal $<$ Prikou $>$, que podría referirse a un equivalente del bri(u)gu irlandés antiguo ('landowner, hospitaller', de $\left.* b^{h} r g^{\text {'h}}-\bar{o} t-s^{59}\right)$, puede corresponder tanto al tipo onomástico BRIGIOS / BRIGIUS como al tipo BRIGO / BRIGIO.

- Si además pensamos no tanto en la dimensión geográfica del lepóntico, sino más bien en su aspecto lingüístico, es decir en el hecho de que constituye un desarrollo particular del celta del $2^{\circ}$ periodo, podemos añadir al listado de genitivos temáticos también los grafitos Kelešu y Košio sobre dos cuencos cerámicos hallados en Casalandri-Isola Rizza (Verona, CIT 264 y 265): Kelešu a partir de *Kel(e)t-yo-s, a comparar con los temas en -(y)o Keltie $<*$ Kelt-yo-s y CELTIUS / CELTIA al lado de CELTUS ; en él se aprecia la misma epéntesis que se ha desarrollado en NPP como Eluveitie (célt. *(H)éluet-yo-s) y en NLL como MONTEM BERIGIEMAM (acus. de célt. *Bérg-yo-mā), junto con la asibilación del nexo ty $\mathrm{V}^{60}$. Košio es evidentemente una forma que se ha desarrollado a partir del mismo *Kosyos con declinación temática implícito en el $<$ Kosioiso $>$ de Castelletto Ticino con alomorfo genitival *-osyo (se trata en realidad de [Kos:yoiso], dado que este genitivo se deja explicar satisfactoriamente sólo a partir de *Kossyosyo ${ }^{61}$ ), en el femenino Koiša, que también nos enseña la misma prepalatalización céltica occidental, y en COSSIUS / COSSIA a partir de Cossus.

- De ser correcta la lectura en CIT 299, se podría incluir entre los candidatos a genitivos también el PIRIXIO grabado en un puñal de tipo céltico hallado en Verzegnis (Udine), si es que continua una forma *Brixio.

- Por otro lado, no se puede decir nada acerca de la inscripción CIT 107 sobre una pátera de Somma Lombardo, dado que la secuencia <ateuloipitus $>$ parece contener una forma verbal en vez de ser una simple fórmula onomástica.

3.3. A pesar de que los argumentos citados siguen siendo válidos, dado que los nuevos hallazgos tan solo han debilitado algo el primero de los morfológicos, o sea el $\mathrm{IIa}^{62}$, lamentablemente muchos estudiosos, después de un primer período de interés general y discusión constructiva de la nueva hipótesis, parecen haber vuelto a la explicación más antigua, que consideraba, por falta de ideas, todas las formas lepónticas de NPP en - $u$ /-io como nominativos de tema en nasal, unos de ellos empleados incongruentemente con función patronímica ${ }^{63}$.

Una de las razones para rechazar la propuesta de J. de Hoz fue, para algunos estudiosos, la existencia de otros dos tipos de genitivo temático en la Céltica italiana -incluso en el mismo corpus más o menos lepóntico ${ }^{64}$, aunque este argumento carece

\footnotetext{
${ }^{59}$ Cf. DBSt (1997/2007A: 163 n. 133) y NWÄI: 163, 171 y 438, con discusión de la bibliografía anterior.

${ }^{60}$ Cf. DBSt (2002/09: 163 y 177ss.).

${ }^{61}$ Por otro lado, *Kosyos sin geminación hubiera proporcionado **Koisoiso, y el genitivo de *Gostyos hubiera sido **Gozioiso, cf. DBSt (2002/09: 179). Para la anticipación de la palatalidad v. abajo la n. 67.

${ }^{62} \mathrm{Se}$ advertirá, de cualquier forma, que la lista de seguros y posibles temas en - $n$ - lepónticos ofrecida por Solinas (2005: 564s.) no es — como subraya ella misma - completa; además, las formas en -oni que allí se incluyen se analizarán mejor — según ya comentaron, entre otros, Eska y Wallace (2001B) — como dativos de temas en nasal, dado que concuerdan con formas patronímicas en -alui.

${ }^{63} \mathrm{~V}$. la discusión al final de este párrafo.

${ }^{64}$ Digo «más o menos» por la razones expuestas arriba en el § 3.1(a).
} 
ahora de pertinencia ante los tres morfemas de genitivo temático empleados en itáli$\mathrm{co}^{65}$ y los por lo menos cuatro aprovechados en anatólico ${ }^{66}$.

Por otra parte, es oportuno subrayar que, hasta la fecha, ni los genitivos en -oiso ni los en $-i$ han aparecido en la misma inscripción al lado de un genitivo en $-u$. En particular, el genitivo en -oiso, procedente de un morfema *-osyo no por metátesis, sino por efecto de una anticipación de la palatalidad cuando en el medio había una consonante simple $(\mathrm{ViCV}<* \mathrm{VCyV})^{67}$, se atestigua en documentos muy antiguos y - al parecer- en indicaciones de pertenencia ${ }^{68}$, mientras que el morfema $-u$ se utiliza más a menudo, y para indicar tanto posesión como procedencia (v. la n. 3 arriba).

Con las mismas finalidades se atestiguan también los genitivos en $-i$, pero éstos son mucho menos frecuentes y a menudo se hallan en inscripciones más modernas, ya sea con respecto a su cronología absoluta, que es en prevalencia La Tène, o al menos con respecto a su estilo, dado que no aparecen nunca en conexión con patronímicos ni preceden al idiónimo principal, sino que siempre van detrás de él ${ }^{69}$ : cf. Alkouinos Aškoneti 'Alkóvindos, [hijo] de Adgónnetos' en Stabio frente al orden más arcaico Aškonetio Pianu en Brisino (cf. la traducción en § 3.1 (I) arriba). Con respecto al Ki甲isi, genitivo en $-i$ de nombre personal posiblemente céltico, que aparece sobre un fragmento cerámico perteneciente a la facies de Golasecca IIIA ${ }^{70}$, recordaremos que en lingüística hablamos siempre en términos de cronología relativa y raras veces de cronología absoluta, como lo ilustra p.ej. el hecho de que - aunque el paso de $* k^{w}$ a $p$ se atestigua ya para el siglo IV a.C. ${ }^{71}$ — en textos inscritos con alfabeto latino siga apareciendo la forma ATECVA sin labializar, continuación de la antigua forma Atekua típica del corpus lepóntico stricto sensu en alfabeto de Lugano, frente a la variante labializada de textos incluso más antiguos, entre otros en alfabeto griego ${ }^{72}$.

En lo que respecta a este tipo de críticas, es necesario adelantar que bajo la nueva perspectiva aplicada a la declinación indoeuropea — perspectiva que permite explicar el origen del genitivo temático en general, algo que constituía uno de los mayores problemas del indoeuropeo reconstruido, como lógico desarrollo de un proceso muy simple ${ }^{73}$ - el escenario ya no es el — todavía asumido por unos críticos- de un ge-

${ }^{65}$ Es decir $*_{-} \bar{\imath}$, *-osyo y el morfema -eis que — según se muestra en DBSt (2000B: 53) — tiene que haber reemplazado el genitivo en $-\bar{\imath}$ en los dialectos del grupo itálico que llamamos «nucleares».

${ }^{66}$ Es decir *-oso- y *-osyo- además del -aš anatólico y de varios sufijos de pertenencia, cf. Melchert (UCLA 1 y 2) con bibliografía; el mismo autor niega la utilización del genitivo de plural para el singular en hitita antiguo. Rieken (2009: 42); DBSt (2001/03: 49s.).

${ }^{67}$ Se trata de una anticipación bastante frecuente en el celta occidental, cf. DBSt (2002/09) con la bibliografía anterior.

${ }^{68}$ Éste es el caso no solamente de $<$ Xosioiso $>$ en Castelletto ( $\$ 3.2$ arriba) y de los varios $<$ Plioiso $>$ en Rondineto, sino también de $<$ Kaialoiso $>$ en Oderzo: '[Tumba] de (-oiso) el hijo de (-alo-) Gaios (Kaio-)', cf. DBSt (2002/09: 179s.). Para otro posible genitivo en -oiso cf. Gambari (2009: 72 con la n.1). Acerca de *-osyo como morfema de genitivo utilizado en particular en idiónimos cf. Watkins (1998/99=2008: 997s.).

${ }^{69}$ Cf. ya DBSt (1992/93: 48s.).

${ }^{70}$ Interpretación proporcionada por Rubat Borel (2005: 26) para la inscripción CIT 223, procedente de Capriate (BG).

${ }^{71}$ Cf. Falileyev (2008: 10).

${ }^{72}$ V. arriba, § 3.1 (III.a) con la n. 40.

${ }^{73}$ Explicado abajo en la sección 4. 
nitivo en -oiso, supuestamente arcaico y polivalente, al cual sustituyera, por razones desconocidas, un extraño morfema genitival $-u$ extraído del ablativo, sino más bien de una muy antigua desinencia ie. ${ }^{*}-\bar{o} d$ que se empleaba - entre otros como $-u$ en dialectos del celta - indiferentemente para genitivo y ablativo al mismo tiempo, cosa que, con el paso de los siglos, empezó a considerarse ambigua, de manera que se procedió a sustituir paulatinamente dicho morfema, en unas de sus funciones o en todas, dependiendo de las áreas.

Otros estudiosos no acogieron la hipótesis de J. de Hoz por no aceptar la explicación ablatival del genitivo celtibérico (arriba en la sección 2) - a veces por haber propuesto ellos mismos, con anterioridad, una explicación diferente ${ }^{74}$.

El autor de un artículo bastante reciente se empeña, pues, en defender la importancia y frecuencia de los temas en nasal en indoeuropeo como si De Hoz no hubiese concedido él mismo que unas cuantas de las formas en $-u$ son temas en $-n^{-{ }^{75}}$. En el mismo artículo se critica una supuesta propensión hacia los patronímicos, revelando de esta manera haber mal comprendido la hipótesis genitival con respecto al lepóntico, que sí contempla toda una serie de funciones diferentes para las formas en $-u$. De hecho, con respecto a la interpretación — todavía muy de moda - de todas las formas onomásticas en $-u$ del entorno de Golasecca como temas en $-n$ en parte patronímicos, el mismo De Hoz (1990: 320) señaló la improbabilidad de que un sufijo productivo para formar idiónimos fuese (¡más tarde!) empleado para formar patronímicos, dado que se hubiera introducido sin necesidad alguna una ambigüedad en las secuencias de $\{$ Patroním. + NP\}y $\{\mathrm{NP}+$ Patroním. $\}$. También se advirtió que, de ser patronímicas todas las formas en $-u$, sería muy dificil explicar tanto las fórmulas onomásticas con dos componentes que terminan en $-u /$-io como las numerosas inscripciones que tan sólo consisten de un único nombre en $-u^{76}$; es verdad que estas últimas podrían representar simples y normales temas en $-n$, es decir no patronímicos, pero sería extraño ver tantos nominativos en piezas de cerámica, dado que los nombres personales pertenecientes a otras declinaciones aparecen aprovechados también en genitivo.

Nadie emprendió una refutación del aspecto filológico de la hipótesis de J. de Hoz: los argumentos estructurales enumerados arriba en $\S 3.1$ pasaron sencillamente desapercibidos. Tampoco nadie buscó una alternativa válida para explicar las formas en -io: los que no aceptan la hipótesis de un genitivo las consideran mayoritariamente nominativos asigmáticos de temas en -yo, lo que choca con la regular preservación de las $s$, tanto finales como intervocálicas, en el corpus lepóntico y en general en todo el galo cisalpino.

En general, la posibilidad inherente a la hipótesis genitival lepóntica desconcertó a muchos autores, de manera que unos la tildaron sin más de improbable y otros la declararon una teoría posible, pero aún por demostrar.

\footnotetext{
${ }^{74} \mathrm{~A}$ este respecto, se tendrá que subrayar que las interpretaciones genitivales dadas para celtibérico y lepóntico, aunque perfectamente paralelas, son totalmente independientes una de otra: ni surgieron para apoyarse mutuamente, ni se necesitan reciprocamente.

${ }^{75}$ Entre otros, en el evidente caso de $\mathrm{Deu}$, analizado arriba en el $\S 3.1$ (IIIa). Acerca de los temas célticos en nasal cf. NWÄI: 99-120, DBSt (1998/07A: 147-150), Wedenig \& ead. (2005/07: 622s.), todos a añadir a Vallejo Ruiz (2004).

${ }^{76}$ DBSt (1992/93: 48).
} 


\section{EL GENITIVO-ABLATIVO SINGULAR EN INDOEUROPEO}

4.1. Si abordamos la cuestión bajo una perspectiva más general ${ }^{77}$ y no limitada al entorno céltico, nos daremos cuenta de que no hay razones de ningún tipo para pensar que los temas en -o- tengan que haber distinguido su genitivo y su ablativo desde el comienzo de la existencia de una declinación del singular ${ }^{78}-\mathrm{y}$ menos aún si consideramos que todas las demás declinaciones, es decir, tanto las más arcaicas como los temas en $-\bar{a}$, más modernos que los en -o-, utilizaban sólo un único morfema para expresar relaciones sintácticas ablativales y genitivales.

Es más, la reconstrucción de una sola forma, sin diferenciar, de genitivo-ablativo para t o d a s las declinaciones encaja muy bien en la reconstrucción más moderna del sistema nominal indoeuropeo, que postula para la época más antigua tan sólo la existencia - en singular - de los casos dichos 'gramaticales', o sea nominativo, dativo y acusativo, a los cuales se habría añadido el genitivo - iy en nuestra opinión ablativo! 'adjetival' mucho antes de que se darrollaran los casos específicamente 'locales' ${ }^{\text {'9 }}$.

4.2. Sin embargo, la cuestión del genitivo fue más bien uno de los casos donde el descubrimiento sólo paulatino de los datos indoeuropeos condicionó su reconstrucción: al principio, de todas las lenguas que preservan restos del genitivo-ablativo temático singular, sólo se conocían lituano, letón y eslavo, lo que llevó a pensar que el empleo del mismo morfema para los dos casos de los temas en -o- debió ser una innovación particular de los territorios nororientales, que no se habría extendido ni siquiera al báltico suroccidental. Y cuando se empezaron a conocer las variedades de griego más arcaicas, se consideró simplemente que dicha innovación había tenido una extensión algo mayor, y así sucesivamente cada vez que se identificaban más ejemplos posibles de la misma coincidencia entre el morfema de genitivo y de ablativo singular temático. Tenía, de hecho, razón W.P. Lehmann en resaltar que en general se presta menos atención a los restos morfológicos que a los fonológicos, pese a ser justo la identificación de restos la llave para descubrir la estructura más antigua del indoeuropeo ${ }^{80}$.

4.3. Por otro lado, si nos libramos de opiniones preconcebidas y ponemos en la balanza todos los datos al mismo tiempo, es natural ver en la igualdad del morfema no una innovación, sino un arcaísmo compartido con todas las demás declinaciones, mientras que la innovación reside, evidentemente, en la gran variedad de morfemas que en las etapas intermedias del indoeuropeo se emplearon para distinguir el genitivo ${ }^{81}$, más a menudo referido a humanos y/o animados. Es, por lo tanto, natural que la necesidad de establecer una distinción surgiera a partir del ámbito de los temas en

${ }^{77}$ Como, de hecho, hicimos hace unos años, cf. DBSt (1998/2007: 166-169) y (2001/03: 46ss.), donde los diferentes aspectos de la presente teoría son explicados en más detalle.

${ }^{78}$ En este sentido ya Porzig pace Lehmann (1981: 185).

${ }^{79}$ Cf. W.P. Lehmann, entre otros (1993: 154), (1999: 79s.) y (2002: 185).

${ }^{80}$ Lehmann (1993: 151) y, respectivamente, (1999: 71).

${ }^{81}$ Cf. también Lehmann (1981: 185). 
$-o^{8}{ }^{82}$, si consideramos que ellos incluían muchos nombres personales y otras designaciones de seres animados y humanos. Dicha suposición parece respaldada por el hecho de que, para desambiguar el genitivo, se emplearon, entre otros, un conglomerado del nominativo masculino singular temático y del pronombre relativo correspondiente (ipropio del indoeuropeo intermedio!), o sea $*_{-o-s}+y o(-s)>*_{-o s y o-}{ }^{83}$, el morfema de genitivo del pronombre personal masculino singular, adjetivos patronímicos y otros derivativos con morfemas de pertenencia ${ }^{84}$.

Es más, mientras que el morfema $-\bar{o} d$, cuyo origen se tendrá probablemente que buscar en un sintagma posposicional del indoeuropeo antiguo, continuó siendo empleado en ablativos y adverbios de la mayoría de los dialectos indeouropeos ${ }^{85}$, el genitivo temático en - $\bar{d} d$ se preservó en particular en lenguas conocidas por el marcado arcaísmo de su declinación, como el eslavo y el báltico oriental ${ }^{86}$.

También las variedades más arcaicas del griego (en particular el micénico y el chipriota) preservaron en su genitivo el morfema -ōd originario del antiguo genitivoablativo, junto con el tracio, dacio, parte del frigio y - muy probablemente- las variedades más arcaicas del celta, una familia en general muy arcaica con respecto al indeouropeo común, es decir que empezó a separarse muy pronto del indeouropeo ${ }^{87}$.

${ }^{82}$ Con el desciframiento del hitita, se llegó a pensar que el morfema -aš que aparece en el genitivo singular de los temas en -o-del periodo clásico podía ser la marca indoeuropea original, que todas las lenguas indoeuropeas, tanto las anatólicas como las demás, habrían sustituido después; pero ya Neu (1979: 186) consideraba dicha suposición «unwahrscheinlich» ante la diversidad de formaciones de genitivo indoeuropeas. Además, ¿por qué los temas indoeuropeos en -o-, pese a ser más recientes de los demás, a la hora de crear un nuevo morfema especial de genitivo para obviar la ambigüedad con un caso tan periférico como el ablativo (ambigüedad tolerada por otro lado en el singular de todas las demás declinaciones), habrían introducido una nueva ambigüedad, esta vez con algo todavía más central como el nominativo de singular? ¿Y cómo es que el genitivo en cuestión no se halla en el corpus más antiguo? Parecería, por lo tanto, más lógico pensar que se tratara del mismo nominativo singular aprovechado en yuxtaposición para expresar un genitivo diferente del ablativo.

${ }^{83}$ Acerca de la utilización de *-osyo- para nombres de persona véase la bibliografía arriba en la n. 68 , y acerca de su origen en particular Lehmann (1981).

${ }^{84}$ Siendo los otros medios la repetición del nominativo singular (varias lenguas anatólicas), el aprovechamiento de las desinencias de genitivo de otros temas (tocario, dialectos nucleares del grupo itálico) y números (¿hitita antiguo?), y también de las desinencias de otros casos (términos de parentesco y nombres propios en tocario).

${ }^{85}$ Cf. entre otros Fortson (2007: 114, § 6.49). Acerca de la continuación del morfema en anatólico, donde se preservó en la expresión del instrumental hitita, véase ahora Melchert y Oettinger (2009). Hackstein (2004: 94s.).

${ }^{86}$ Igartua (2003) ha vuelto a evaluar todos los datos que proceden de dichas ramas, llegando a la conclusión de que el genitivo singular en *-o $d$ no constituye una prueba en favor de una unidad balto-eslava. Además, ante la evidencia recogida por DBSt (2001/03), «the hypothesis of a Balto-Slavic isomorph concerning the origin of the thematic gen.sg. [...] ought to be entirely abandoned, since the retention of features cannot be taken into account for grouping purposes» (Igartua, 2003: 60).

${ }^{87} \mathrm{Cf}$. las varias isoglosas enumeradas por DBSt (2001/03: 31-39). Es interesante notar que el genitivo micénico en $-\bar{o}$ es mayoritariamente de tipo partitivo, cf. op.cit.: 46 y Bartoňek (2003: 304). 


\section{VIEJAS Y NUEVAS CONTINUACIONES CÉLTICAS DEL GENITIVO- ABLATIVO SINGULAR TEMÁTICO PROTOINDOEUROPEO}

5.1. Si ahora volvemos a acercarnos a la subfamilia céltica, veremos que se pueden rastrear incluso más huellas de lo que suponemos fue el genitivo-ablativo indiferenciado de los temas en - $O$ - en el indoeuropeo antiguo.

En la sección 2, hemos visto su preservación funcional en celtibérico, pese a la innovación fonética que acabó abreviando con regularidad la $-\bar{o} \#$ en $-o \#^{88}$. Además hay - como advertí ya en otros estudios ${ }^{89}$ — por lo menos un caso donde el morfema celtibérico en cuestión se documenta, al parecer, fuera del corpus epicórico hallado en la propia Celtiberia: se trata de una moneda, fechada en el siglo II a.C. y clasificada hasta ahora como gala $(R I G$-IV-157) a pesar de los rasgos lingüísticos —más bien celtibéricos que galos - de su leyenda EIQITIAICO, donde, además del genitivo temático en $-o$, se aprecian la preservación de la labiovelar sorda, el digrama $<$ ei $>$ para expresar [e] y el característico morfema -aico- a partir del conglutinado sufixal *-āk-yo-.

Se notará que en celtibérico la función ablativa del genitivo-ablativo protoindoeuropeo se puede apreciar todavía, tanto en el caso de los temas en $-\bar{a}$ discutido arriba (§ 2.1) y visible en muchos pactos de hospitalidad donde topónimos caracterizados por los alomorfos -as / - az tienen valor de ablativo, como en el caso de los temas en -o-: cf. el complemento de agente en la frase S.e.ki.l.a.ko: ... :a.ta 'estipulada (ata $<*$ âgtā) p o r Segilakos', de la tésera de Arecoranda K.0.11, y los complementos de argumento en la secuencia To.ko.i.to.s.ku.e: S.a.r.n.i.ki.o.ku.e, que se repite, con el sentido de «A c e r c a d e Togotis y a c e r c a d e Sarnicios», en los $\S \S$ II y VII del 1. ${ }^{\text {er }}$ bronce de Botorrita ${ }^{90}$.

5.2. En Italia, la función de ablativo se aprecia específicamente en el miliario citado arriba con inscripción Mešiolano (§ 2.2), mientras que - como hemos visto en la sección 3- el genitivo-ablativo en - $(y) o /-u$ se emplea en indicaciones de posesión ${ }^{91} \mathrm{y}$ de origen tanto en inscripciones lepónticas stricto sensu como en otras procedentes de los territorios cisalpinos más orientales.

Más huellas de un genitivo-ablativo temático inalterado parecen conservarse en el celta de tipo ligur, es decir de Liguria: en las tres estelas con inscripción que se conocen - fechadas entre los siglos VII y VI a.C. y procedentes de Filetto, Bigliolo y Zignago (CIT 272-274) - , las fórmulas onomásticas empiezan por un elemento en - $u$ que precede al nominativo singular de un idiónimo y que podría indicar un genitivo de paternidad; se trata de Vezaru o Uvezaru, Veme日u у Mezu, a los cuales corresponden Abus, Vis у Nemuśus ${ }^{92}$.

\footnotetext{
${ }^{88}$ Paso que, por lo visto, parece haberse realizado después del Celtibérico arcaico.

${ }^{89}$ Entre otros DBSt (2005/07: 153).

${ }^{90}$ Cf., para el primer ejemplo, DBSt (2000A: 187) en la estela de Gorrochategui (1990) y, para el segundo, la nueva interpretación en ead. (2008/10: 125s., 133s., 136) y (2009: 689).

${ }^{91}$ Sin embargo, el morfema -oiso con el cual dicho genitivo-ablativo alterna en este tipo de contextos (§ 3.3 arriba) parece ser una continuación céltica occidental del nuevo 'genitivo pronominalizado' ${ }_{\text {-os-yo, }}$ adquirido del cercano mundo itálico.

${ }_{92} \mathrm{De} * A b(b) o s, *$ Wik-s 'Combatiente' y *Némausos, mientras que las supuestas indicaciones de paternidad podrían quizás retrotraerse a algo como *Wedya-ro-s o *U(p)e-dya-ro-s, *U(p)o-met-o-s, *Med-yo-s; cf. DBSt (2006: 47) y (2005/06: 49).
} 
5.3. En goidélico, encontramos unas formas residuales de genitivo-ablativo temático que se quedaron cristalizadas, con función de propatronímico, en varias fórmulas onomásticas cuando, en los patronímicos, el nuevo genitivo en $*_{-} \bar{l}$ empezó a sustituir las formas en -u originarias. Se trata por un lado del $* m a ́ k k^{w} \bar{o} d^{93}>* m a q q(w) u$ que - perteneciendo a un nivel de lengua muy antiguo - sigue apareciendo como sustantivo invariable $m a c(c) u / m o(c) c u$ en textos del irlandés arcaico y se atestigua incluso antes en ogam como MAQU / MAC(c)U 'de(sde) el hijo' y — amplificada luego con la - $\bar{l}$ del nuevo genitivo singular MAQI- como MOCOI / MUCOI ${ }^{94}$; por otro lado, de corcu (más tarde corco / corca) 'de(sde) la semilla', que se relaciona con coirce 'avena'. Ejemplos de su utilización son las perífrasis propatronímicas Miliuc mocu Boin y su equivalente latinizado Miliuc filius nepotis Buain o, respectivamente, Corcu Ruaid 'de la semilla —o sea descendencia - de Ruad'.

5.4. Otro resto de propatronímico parece hallarse en la fórmula onomástica —en acusativo - del quinto personaje que participa en el conjuro de Chamalières ( $R I G$ L-100). De un análisis más cuidadoso de las fórmulas onomásticas involucradas, las cuales - por las razones que ilustramos en otro lugar ${ }^{95}$ - resultan ser en total seis, aparece de hecho una secuencia Caelion $\bullet$ Pelign(on) $\bullet$ Claudio Pelign(u), traducible como 'Caelius Paelignus, descendiente de Claudius Paelignus', donde Claudio y el apositivo de origen - supuestamente Pelign $(u)$ - preservarían las dos variantes fonéticamente complementarias del genitivo-ablativo céltico arcaico.

5.5. A todas las formas citadas se pueden añadir unas cuantas formas «nuevas» si rastreamos los varios corpora que se conocen como galos. Así, en el calendario de Coligny, transcripción de finales del II siglo d.C. a partir de algo mucho más antiguo, encontramos el morfema arcaico de genitivo-ablativo temático en indicaciones temporales, correspondiendo a lo que se suele describir como un complemento de tiempo en genitivo: se trata de $A_{N A G(A N) T I O,} Q_{U T I O} / C_{U T I O}, O_{G R O N U}$, que significan 'durante el mes Anagantios / Qutios / Ogronos', y así mismo de TIOCOBREXTIO 'en el día o fiesta Tiocobrextios', todos hasta ahora considerados como simples aunque inexplicadas variantes ${ }^{96}$. Se advertirá que para $O_{G R O ́ N}(N) O S$, el 'mes del frío', la forma $O_{G R O N U}$-que no podría ser, además, de ninguna manera una variante abreviada — aparece en el mismo texto con el más moderno genitivo en $-\bar{l}$.

Ante dicha evidencia podemos llegar a pensar que, dentro del polifacético corpus

${ }^{93}$ Una propuesta ligeramente diferente en Ködderitzsch (1992/93: 154-156, con 145s. y 152s.), que cuenta con una etimología cruzada, fonéticamente interesante, con un lexema tracio-céltico.

${ }^{94} \mathrm{Cf}$. ahora también Garrett (1999: 149 con la n. 13), que resalta el aspecto sintáctico de las nuevas formas ogámicas en -I y en -OI. Aquellos estudiosos que rechazaron la reconstrucción ablatival de $m a c(c) u / m o(c) c u$ etc. a causa de la preservación de la vocal final, no han entendido el caracter de fósil de las formas involucradas, subrayado por DBSt (1991A) y (1992/93: 49); cf. also ead. (2010: 105 ss. § II.5).

${ }^{95}$ DBSt (2010: $\left.111 \S 6.2\right)$.

${ }^{96}$ El comentario que suele acompañar dichas formas, del estilo de «Les génitifs en cutio, qutio sont étranges au regard de la grammaire comparée, et on doit donc les regarder comme des formes abrégées» (aquí en las palabras de Delamarre 2003: 133), nos enseña una de las numerosas petitiones principii todavía presentes en los rincones de nuestra disciplina. Cf. RIG III: 265-269 and 324s.; Arenas \& DBSt (2003/e.p.). 
que se suele llamar galo, puede haber más casos, todavía no detectados, de preservación del antiguo morfema en función genitival. Por ejemplo, marcas de propiedad del tipo de

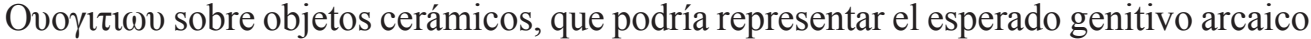
de *Vogitios, no tienen por qué ser necesariamente nominativos de tema en nasal ${ }^{97}$.

Y una leyenda monetal como TASGETIO (RIG-IV-277) ¿representará de verdad una forma abreviada del nombre Tasgetios del caudillo de los Carnutes? ¿o quizás —más apropiadamente - el genitivo arcaico completo y correcto?

Simplemente ambiguas resultarán ahora leyendas monetales como K $\alpha \lambda \varepsilon \tau \varepsilon \delta o v ~ S V L A$ (RIG-IV-91): ¿secuencia de dos nominativos o más bien genitivo de paternidad arcaico del nombre celta ${ }^{*}$ Kaletedos ${ }^{98}$ precediendo el nominativo del idiónimo Sul(l)a? Y de

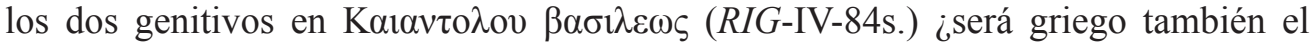
primero o quizás sencillamente indígena?

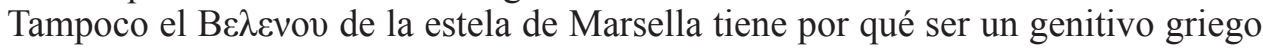
— según postula una de las dos hipótesis en $R I G-\mathrm{G}-24$, que traduce 'de[1 dios] Belenos'-, pudiendo explicarse sencillamente como resto de genitivo céltico arcaico en - $u$.

En cuanto a la problemática inscripción 'galo-griega' de Villelaure hoy lamentablemente perdida ( $R I G-* \mathrm{G}-154)$, su comprensión sintáctica mejora mucho si para por lo menos unas de las formas en - $u \#$ involucradas postulamos una función de origen en vez que de nominativo o dativo. Podemos leer y dividir su texto como:

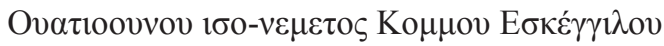

que habría expresado

'De[1 dios] Vatiomnos [es] este-mismo santuario,

[realizado/dedicado] por Commos, hijo-de-Eskengos’,

aunque tampoco se puede descartar la posibilidad que el texto consistiera de dos frases, que leeríamos como

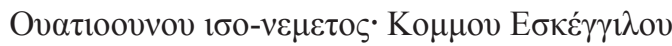

o sea

'De[1 dios] Vatiomnos [es] este-mismo ${ }^{99}$ santuario: Commu [hijo] de Eskengilos'.

En el primer caso, las tres formas en - $u \#$ involucradas serían todas de genitivo, en el segundo tan sólo el teónimo.

${ }^{97}$ Como suponen RIG-G-250-251 y Delamarre (2003: 326s.). También se atestigua un compuesto como Vogitoutos con genitivo Vogitouti (cf. CLI: Index), lo que podría quizás apuntar a un original *Vogitoutios.

${ }_{98}^{98}$ Implicado también por el nominativo latinizado — de tema en nasal- Caletedo de la Narbonense (NPC s.v.).

${ }^{99}$ Con iso(s) < *isto-s como en el išos de Vergiate y en la conjunción correlativa iste_iste 'lo mismo... que...', cf. DBSt $(2008 / 10: 132,137)$ y $(2009: 688,691)$, ambos con bibliografía. 
También la sortija suiza de Vindonissa/Windisch $\left(R I G\right.$ - $\left.^{*} \mathrm{~L}-131\right)$ recobra sentido si entendemos IXUTIOU como genitivo arcaico del nombre del padre (Ixutios) de la abuela (Tocna, posiblemente de *Toncna, aquí en genitivo de procedencia TOCNAE) que regala la sortija a su nieto. La inscripción del anillo reza

\section{TOCNAE IXUTIOU DRUTO AVO MIO}

y es perfectamente traducible como

'[Don] de parte de Tocna, [hija] de Ixutios, para Drutos, mi nieto'.

Concluiremos recordando que, con el tiempo, el $*_{-} \bar{o} d$ del indeouropeo arcaico se fundió en celta con la $-u$ que continuaba la $*_{-}-\bar{o} \#$ final primaria del instrumental temático indoeuropeo. La misma desinencia céltica $-u$ acabó por sustituir, en el dativo singular,

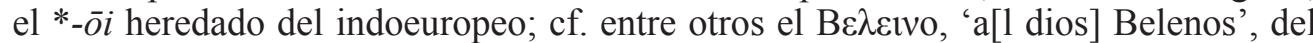
galo arcaico y el más tardío ANVALÓNNACU, 'al lugar — santuario o templo- del dios Anvállos' frente al galo CELICNU 'en el salón’ (RIG-G-128, L-10, L- 51) ${ }^{100}$.

5.6. Los tipos de formas residuales de genitivo-ablativo identificados en los diferentes dialectos célticos encajan, además, - como se aprecia con facilidad en la tabla siguiente — con las fases de desarrollo del celta que ahora conocemos:

\begin{tabular}{|c|c|c|}
\hline \multirow{3}{*}{$\begin{array}{l}\text { I. genitivo- } \\
\text { ablativo } \\
\text { singular } \\
\text { indiferenciado } \\
\text { en cada } \\
\text { declinación }\end{array}$} & en indoeuropeo antiguo & y en protocelta \\
\hline & & $\begin{array}{l}\text { continuado en el } 1 .^{\text {er }} \text { periodo celta, } \\
\text { con el morfema temático abreviado } \\
\text { en celtibérico }\end{array}$ \\
\hline & & $\begin{array}{l}\text { continuado en el } 2 .^{\circ} \text { periodo celta, } \\
\text { con el morfema temático aflorando } \\
\text { en unos cuantos documentos arcaicos }\end{array}$ \\
\hline
\end{tabular}

\begin{tabular}{|c|c|c|c|}
\hline \multirow{3}{*}{$\begin{array}{l}\text { II. genitivo } \\
\text { singular temático } \\
\text { diferenciado } \\
\text { por medio de } \\
\text { varios morfemas }\end{array}$} & $\begin{array}{l}\text { del indoeuropeo } \\
\text { intermedio }\end{array}$ & \multicolumn{2}{|c|}{ y en celta, del $2 .^{\circ}$ periodo en adelante } \\
\hline & & $\begin{array}{l}\text { *-osyo se adopta } \\
\text { en lepóntico en } \\
\text { indicaciones de } \\
\text { posesión }\end{array}$ & $\begin{array}{l}\text { viejo morfema }-u<*_{-} \bar{d} \\
\text { todavía bastante utilizado }\end{array}$ \\
\hline & & $\begin{array}{l}*_{-} \bar{l} \text { se utiliza con } \\
\text { normalidad del } 3 . \text { er } \\
\text { periodo celta en } \\
\text { adelante (goidélico; } \\
\text { galo; britónico) }\end{array}$ & $\begin{array}{l}\text { genitivo-ablativo } \\
\text { temático residual relegado } \\
\text { a funciones marginales } \\
\text { del 3. }{ }^{\text {er }} \text { periodo celta en } \\
\text { adelante (goidélico; galo) }\end{array}$ \\
\hline
\end{tabular}

${ }^{100}$ Cf. DBSt (1998/2007: 161) y (2008: 86) con la bibliografía anterior. 
Finalmente, — como ya adelantamos a finales de la sección 4- de todo el material analizado podemos deducir una vez más un índice de arcaísmo muy alto para el proceso de glotogénesis de lo que se clasifica como subfamilia céltica del indoeuropeo.

\section{BiBLIOGRAFÍA}

Arenas Esteban, J.A. \& P. De Bernardo Stempel (2003/e.p.), «Celtic Dialects and Cultural Contacts in Protohistory: the Italian and Iberian Peninsulas», Études Celtiques 37.

Arenas [Esteban], J.A., P. de Bernardo Stempel, M.C. González \& J. Gorrochategui (2001), «La estela de Retugenos (K.12.1) y el imperativo celtibérico», Emerita 69 (2): 307-318.

BARTOŇEK, A. (2003), Handbuch des mykenischen Griechisch. Heidelberg: C. Winter.

Birkhan, H. (ed. con la colab. de H. Tauber; 2007), Kelten-Einfälle an der Donau. Akten des 4. Symposium deutschsprachiger Keltologinnen und Keltologen (Linz/Donau, Juli 2005). Viena: Österreichische Akademie der Wissenschaften, Phil.-hist. Klasse, Denkschriften n. ${ }^{\circ} 345$.

Burillo Mozota, F. $\left(2008^{2}\right)$, Los Celtíberos: etnias y estados, $2^{\mathrm{a}}$ edición actualizada. Barcelona: Crítica.

Celtes et Gaulois I: 33-56 = De Bernardo Stempel (2005/06).

CIT = Piana Agostinetti, P. (ed.; 2004), Celti d'Italia 2 vols. Roma: Biblioteca di Storia patria, Popoli e civiltà dell'Italia antica 12.

CLI = Raybould, M.E. \& P. Sims-Williams (2007-2009), A Corpus of Latin Inscriptions of the Roman Empire containing Celtic Personal Names. Con Introduction and Supplement to .... Aberystwyth: CMCS.

DBSt véase De Bernardo Stempel, $P$.

De Bernardo Stempel, P. (1990), «Einige Beobachtungen zu idg. /w/ im Keltischen» en A.T.E. Matonis \& D.F. Melia (eds.), Celtic Language, Celtic Culture. A Festschrift for E.P. Hamp, Van Nuys/Calif., Ford \& Bailie: 26-46.

- $\left(1991^{\mathrm{a}}\right)$, «Archaisch Irisch maccu als morphologisches Relikt», Historische Sprachforschung 104 (2): 205-223.

- (1991b), «Die Sprache der altbritannischen Münzlegenden», Zeitschrift für celtische Philologie 44: 36-55.

- (1992/93), «Probleme der relativen Chronologie: nochmals zu idg. *ō im Keltischen» en ROCKel \& ZiMmer (eds.; 1993): 37-56.

- (1998), «Minima Celtica zwischen Sprach- und Kulturgeschichte» en P. Anreiter, L. Bartosiewicz, E. Jerem \& W. Meid (eds.), Man and the Animal World. GS S. Bökönyi, Budapest, Archaeolingua 9: 601-610.

- (1998/2007a), «Le declinazioni nel celtico continentale: innovazioni comuni al gallico e al goidelico?» en LAMBERT \& PinAult (eds.; 2007): 145-179.

- (1998/2007b), «Sull'origine delle sibilanti in celtiberico: una modifica alla teoria di Francisco Villar» en LAMBerT \& Pinault (eds.; 2007): 181-188.

— (1999/2001), «Grafemica e fonologia del celtiberico» en F. Villar \& M. P. Fernández Álvarez (eds.; 2001), Religión, lengua y cultura prerromanas de Hispania. Actas del $8^{\circ}$ Coloquio 
sobre lenguas y culturas prerromanas de la Península Ibérica (Salamanca, mayo de 1999), Salamanca, Universidad: 319-334.

De Bernardo Stempel, P. (2000a), «Celtib. karvo gortika 'favor amicitiae', rita 'ofrecida', monima 'recuerdo' y los formularios de las inscripciones celtibéricas», Veleia 17: 183-189.

- (2000b), «Kernitalisch, Latein, Venetisch: ein Etappenmodell» en M. Ofitsch \& Ch. Zinko (eds.), 125 Jahre Indogermanistik in Graz, Graz, Leykam: 47-70.

- (2001/03), «Der Beitrag des Keltischen zur Rekonstruktion des indogermanischen Nomens» en E. Tichy, D.S. Wodtko \& B. Irslinger (eds.; 2003), Indogermanisches Nomen. Akten der Arbeitstagung der Indogermanischen Gesellschaft (Freiburg/Br., September 2001), Bremen, Hempen: 31-50.

- (2002), «Centro y áreas laterales: la formación del celtibérico sobre el fondo del celta peninsular hispano», Palaeohispanica 2: 89-132.

- (2002/09), «La ricostruzione del celtico d'Italia sulla base dell'onomastica antica» en Poccetti (ed.; 2009): 153-192.

- (2003), «Continental Celtic ollo : Early Welsh (h)ol(l), Olwen and Culhwch», Cambrian Medieval Celtic Studies 46: 119-127.

- (2004), «Cib. oboi 'sea eso'... alaboi 'o bien sea' ... : Morfosintaxis céltica en el Bronce de Córtono (K.0.7, Ll. 1-2)», Palaeohispanica 4: 135-151.

- (2004/05), «Tratamiento y notación de las silbantes en celtibérico: cronología relativa del desarrollo paulatino visible en inscripciones y monedas» en J. Velaza Frías, F. Beltrán Lloris \& C. Jordán Cólera (eds.; 2005), Acta Palaeohispanica IX (Barcelona, octubre de 2004), Zaragoza \& Barcelona = Palaeohispanica 5: 539-563.

- (2005/06), «Language and the Historiography of Celtic-Speaking Peoples» en V. Guichard et al. (coord.), Celtes et Gaulois: L'archéologie face à l'histoire. Tables-rondes internationales 2005, vol. I: S. Rieckhoff (ed.; 2006), Celtes et Gaulois dans l'histoire, l'historiographie et l'idéologie moderne (Leipzig, juin 2005), Glux-en-Glenne, Bibracte n. ${ }^{\circ}$ 12/1: 33-56.

- (2005/07), «Varietäten des Keltischen auf der Iberischen Halbinsel: Neue Evidenzen» en BIRKHAN (ed.; 2007): 149-162.

- (2006), «From Ligury to Spain: Unaccented *yo > (y)e in Narbonensic Votives ('Gaulish' $\delta \varepsilon \kappa \alpha \nu \tau \varepsilon \mu)$, Hispanic coins ('Iberian' -(sk)en) and some Theonyms», Palaeohispanica 6: 45-58.

- (2007/08), «I nomi teoforici del celtico antico. Individuazione, classificazione, divinità venerate e cronologia relativa» en A. Sartori (ed.; 2008), Dedicanti e cultores. VIII Workshop F.E.R.C.AN. (Gargnano del Garda, maggio 2007), Milán, Quaderni di Acme, n. ${ }^{\circ} 104: 73-104$.

- (2008), «I 'compagni’ celtici», Études Celtiques 36: 85-88.

- (2008/10), «La ley del 1. ${ }^{\text {er }}$ Bronce de Botorrita: Uso agropecuario de un encinar sagrado» en F. Burillo Mozota (ed.; 2010), VI Simposio sobre Celtíberos: Ritos y Mitos, Zaragoza, Centro de Estudios Celtibéricos \& Fundación Segeda: 123-145.

- (2008/e.p.), «Il testo pregallico della stele di Vercelli nel contesto delle lingue celtiche» en G. Cantino-Wataghin, F.M. Gambari \& C. Colombara (eds.), Finem dare: il confine tra sacro, profano e immaginario. A margine della stele bilingue del Museo Leone di Vercelli [Convegno Vercelli, maggio 2008], Vercelli, Ed. Mercurio.

- (2009), «La gramática celtibérica del primer bronce de Botorrita: nuevos resultados» en F. Beltrán, J. d’Encarnação, A. Guerra \& C. Jordán (eds.), Acta Palaeohispanica X (Lisboa, 
Fevreiro de 2009), Zaragoza 2009: Fundación Fernando el Católico = Palaeohispanica 9: 683-699.

De Bernardo Stempel, P. (2009/10), «Die Geminaten des Kontinentalkeltischen» en K. Stüber, Th. Zehnder, D. Bachmann (eds.), Akten des 5. Symposiums deutschsprachiger Keltologen (Zürich, September 2009), Viena: Keltische Forschungen - Allgemeine Buchreihe Band 1: 65-87).

- (2010), «Zur Interpretation keltischer Inschriften im Lichte indogermanischer Namenformeln», Incontri Linguistici 33: 87-123 [Continuación en vol. 34/2011, e.p.].

- (en prep.). «Post-tonic Vowels in Continental Celtic».

De Bernardo Stempel, P., C. Sanz Mínguez \& F. Romero Carnicero (2010), «Nueva fusayola con inscripción en signario celtibérico de la necrópolis vaccea de Las Ruedas de Pintia», Palaeohispanica 10: 405-426.

De Hoz, J. (1983/86), «La epigrafía celtibérica» en G. Fatás (ed.; 1986), Reunión sobre epigrafia hispánica de época romano-republicana (Zaragoza 1983), Zaragoza 1986, Institución Fernando el Católico: 43-103.

- (1990), «El genitivo céltico de los temas en -o-: El testimonio lepóntico» en VILLAR (ed.): 315-329.

- (2005), «La lengua y la escritura celtibéricas» en A. Jimeno Martínez (ed.), Celtíberos tras la estela de Numancia, Soria, Junta de Castilla y León: 417-426.

Delamarre, X. (2003), Dictionnaire de la langue gauloise: Une approche linguistique du vieux-celtique continental, 2. édition revue et augmentée. París: Errance.

EsKA, J.F. (1989), «The Verbal Desinence -Tus in the Hispano-Celtic Inscription of Botorrita», Zeitschrift für celtische Philologie 43: 214-222.

- (1995), «Observations on the thematic genitive singular in Lepontic and Hispano-Celtic» en J.F. Eska, R.G. Gruffydd \& N. Jacobs (eds.), Hispano-Gallo-Brittonica. Essays in honour of Professor D.E. Evans, Cardiff, University Press: 33-46.

- (2008), «Continental Celtic», en R.D. Woodard (ed.), The Ancient Languages of Europe, Cambridge, University Press: 165-188.

EsKA, J.F. \& R.E. WALlaCe (2001a), «Remarks on the thematic genitive singular in ancient Italy and related matters», Incontri Linguistici 24: 77-97.

- (2001b), «A syncretism in fieri in early Celtic», Indogermanische Forschungen 106: 229-240.

Falileyev, A.I. (2008), Le Vieux-Gallois. Potsdam: Université.

Fleuriot, L. (1981), «A propos de deux inscriptions gauloises, formes verbales celtiques», Études celtiques 18: 89-107.

Fortson IV, B.W. (2007), Indo-European Language and Culture: an introduction, 6th reprint of the1st ed. 2004, Malden/MA et.al.: Blackwell, Texbooks in Linguistics 19.

GAmbari, F.M. (2009), «L'épigraphie en langue celtique de la Cisalpine» en Chr. Lorre \& V. Cicolani (eds.), Passeurs des Alpes: la culture de Golasecca dans le circuit des échanges européens, Catalogue de l'exposition en Saint-Germain-en-Laye (Novembre 2009), París, Réunion des Musées Nationaux: 71-73.

Garrett, A. (1999), «On the Prosodic Phonology of Ogam Irish», Ériu 50: 139-160.

GipPERT, J. (2004), Reseña de: NWÄI; en Beiträge zur Namenforschung N.F. 39 (1): 118-125.

GonzÁlez Rodríguez, M.C. (1986), Las unidades organizativas indígenas del área indoeuropea de Hispania. Vitoria-Gasteiz, Universidad del País Vasco, Anejos de Veleia, n. ${ }^{\circ} 2$. 
Gorrochategui, J. (1990), «Consideraciones sobre la fórmula onomástica y la expresión del origen en algunos textos celtibéricos menores» en VILLAR (ed.): 291-312.

Gorrochategui, J. (1997), «Die Crux des Keltiberischen», Zeitschrift für celtische Philologie 49-50: $250-272$.

Gusmani, R. (2005), Reseña de: Indogermanisches Nomen. Derivation, Flexion und Ablaut, Bremen 2003: Hempen; en Incontri Linguistici 28: 219.

Hackstein, O. (2004), Reseña de: G. Carling, Die Funktionen der lokalen Kasus im Tocharischen. Berlin \& New York 2000: de Gruyter; en Kratylos 49: 91-97.

Igartua, I. (2003), «On the Indo-European Thematic Genitive Singular and the Balto-Slavic Isomorph», Studia Indogermanica Lodziensia 5: 51-64.

IsaAC, G.R. (2002), «The Celtiberian Alphabetic Signs San and Sigma and the Ablative Singular», Studia Celtica 36: 1-20.

JordÁn Cólera, C. (2003), «Acerca del ablativo que aparece en las téseras de hospitalidad celtibéricas», Palaeohispanica 3: 113-127.

- (2004), Celtibérico. Zaragoza, Universidad, Monografías de filología griega, n. ${ }^{\circ} 16$.

Ködderitzsch, R. (1992/93), «Keltisch und Thrakisch» en Rockel \& Zimmer (eds.; 1993): 139-157.

Lambert, P.-Y. \& G.-J. Pinault (eds.; 2007), Gaulois et Celtique continental [Colloquium Clermont-Ferrand, mai 1998]. Ginevra: Droz, Hautes études du monde gréco-romain n. ${ }^{\circ} 39$.

Lehmann, W.P. (1981), "The Genitive Singular Ending in -syo: How an Indo-Europeanist works» en Y. Arbeitman (ed.), Bono homini donum: Essays in Historical Linguistics in Memory of J.A. Kerns, Amsterdam, Benjamins: 179-188.

- (1993), Theoretical Bases of Indo-European Linguistics. London: Routledge.

— (1999), «Towards a History of Early Indo-European», Diachronica 16 (1): 67-95.

- (2002), Pre-Indo-European. Washington: Institute for the Study of Man, JIES Monograph n. ${ }^{\circ} 41$.

LeJeune, M. (1971), Lepontica . Paris: Les Belles Lettres = «Documents gaulois et para-gaulois de Cisalpine», Études celtiques 12 (2): 337-500.

$L K A=$ Urban, O. (ed. et alii), Lexikon zur keltischen Archäologie. Viena: Österreichische Akademie der Wissenschaften (en prensa).

Matasovič, R. (2009), Etymological Dictionary of Proto-Celtic. Leiden y Boston: Brill.

MeID, W. (2000), «Forschungsbericht Altkeltische Sprachen: Teil III», Kratylos 45: 1-28.

— (2002), «Nachträge zum 'Forschungsbericht Altkeltische Sprachen'», Kratylos 47: 23-29.

- (2005/08), «Ancient Celtic personal names with religious or cultic significance» en R. Häussler \& A.C. King (eds.; 2008), Continuity and Innovation in Religion in the Roman West [FERCAN-Workshop London, April 2005] = Journal of Roman Archaeology Suppl. vol. 67 (2): 87-89.

Melchert, H.C. (ucla 1), «Genitive Case and Possessive Adjective in Anatolian», 12pp.; pdf en www.linguistics.ucla.edu/people/Melchert/.

- (uClA 2), «Further Thoughts on Carian Nominal Inflection», 11pp.; pdf en www.linguistics. ucla.edu/people/Melchert/.

Melchert, H.C. \& N. Oettinger (2009), «Ablativ und Instrumental im Hethitischen und Indogermanischen. Ein Beitrag zur relativen Chronologie», Incontri Linguistici 32: 53-73.

Morand, A. (2000), «Sulla edizione dei testi epigrafici celtici d'Italia», Rivista di Studi Liguri 66: 5-21. 
MotтA, F. (2000), «La documentazione epigrafica e linguistica», en R.C. de Marinis \& S. Biaggio Simona, I Leponti tra mito e realtà, vol. 2, Locarno: Armando Dadò: 181-222.

MotтA, F. (2002/09), «Tipologie dell'onomastica personale celtica nell'Italia antica» en Poccetti (ed.; 2009): 295-318.

Neu, E. (1979), «Einige Überlegungen zu den hethitischen Kasusendungen» en id. \& W. Meid (eds.) Hethitisch und Indogermanisch, Innsbruck, IBS 25: 177-196.

NPC = Delamarre, X. (2007), Noms de personnes celtiques dans l'épigraphie classique. Paris.

NTS-Index = HARTLEY, B.R. \& B.M. Dickinson (2008-), Names on Terra sigillata. An index of makers'stamps \& signatures on Gallo-Roman Terra sigillata (Samian ware), vols. I-ss., Londres: University, Institute of Classical Studies.

NWÄI = De Bernardo Stempel, P. (1999), Nominale Wortbildung des älteren Irischen: Stammbildung und Derivation, Tubinga: Niemeyer, Buchreihe der ZcP n. ${ }^{\circ} 15$.

Poccetti, P. (ed.; 2009), L'onomastica dell'Italia antica. Aspetti linguistici, storici, culturali, tipologici e classificatori (Atti del Convegno di Roma, novembre 2002), Roma: Collection de l'École Française n. ${ }^{\circ} 413$.

Prosdocimi, A.L. (2002/09), «Note sull'onomastica di Roma e dell'Italia antica» en Poccetti (ed.; 2009): 73-151.

Prosdocimi, A. \& P. Solinas (2005/06), «Celticità linguistica in Italia prima del 400. Documenti e prospettive» en V. Guichard et al. (coord.), Celtes et Gaulois: L'archéologie face à l'histoire. Tables-rondes internationales 2005, vol. II: D. Vitali (ed.; 2006), La Préhistoire des Celtes (Bologna, mai 2005), Glux-en-Glenne, Bibracte n. ${ }^{\circ}$ 12/2: 217-234.

Rieken, E. (2009), «Der Archaismus des Hethitischen: eine Bestandaufnahme», Incontri Linguistici 32: 37-52.

$R I G=$ Duval, P.-M. (coord.; 1985-2002), Recueil des inscriptions gauloises, vols. I-IV. París: CNRS.

Rockel, M. \& S. Zimmer (eds.; 1993), Akten des ersten Symposiums deutschsprachiger Keltologen (Gosen bei Berlin, April 1992). Tübingen: Niemeyer, Buchreihe der ZcP n. ${ }^{\circ} 11$.

Rubat Borel, F. (2005), «Lingue e scritture delle Alpi occidentali prima della romanizzazione», Bulletin d'Études Préhistoriques et Arqueologiques Alpines 16: 9-50.

- (2005/06), «Annexe 2: Nuovi dati per la storia delle lingua celtiche della Cisalpina» en V. Guichard et al. (coord.), Celtes et Gaulois: L'archéologie face à l'histoire. Tables-rondes internationales 2005, vol. II: D. Vitali (ed.; 2006), La Préhistoire des Celtes (Bologna, mai 2005), Glux-en-Glenne, Bibracte n. ${ }^{\circ}$ 12/2: 203-208.

- (2009), «L'apport de l'épigraphie à la connaissance des langues celtiques: le celtique de la Cisalpine» en Chr. Lorre \& V. Cicolani (eds.), Passeurs des Alpes: la culture de Golasecca dans le circuit des échanges européens, Catalogue de l'exposition en Saint-Germain-enLaye (Novembre 2009), París, Réunion des Musées Nationaux: 74-75.

Rubat Borel, F. \& O. Paccolat (2008), «Une inscription lépontique découverte à Argnou, commune d'Ayent VS», Annuaire d'Archéologie Suisse 91: 127-133.

Schмid, K.H. (1977), «Zum Problem des Genetivs der o-Stämme im Baltischen und Slavischen» en F. Scholz, W. Woesler \& P. Gerlinghoff (eds.), Commentationes linguisticae et philologicae Ernesto Dickenmann lustrum claudenti quintum decimum, Heidelberg: 335-344.

- (1995), «Zur relativen Chronologie des Genetivs Singular der $o$-Stämme im Baltischen und Slavischen», en H. Schaller et alii (eds.), Studia linguistica, Festschrift für I. Duridanov, 
Sofia 1995, Universitätsverlag St. Kliment Ohridski = Archiv für bulgarische Philologie 3/ 1999: 159-167.

— (1999), «Rezensionsaufsatz zu VILLAR 1995», Zeitschrift für celtische Philologie 51: 190-202.

SchriJver, P. (1998/2007), «Some common developments of Continental and Insular Celtic» en LAmbert \& Pinault (eds.; 2007): 355-371.

ShiElds, K. Jr. (2005), «On the Indo-European Genitive Suffix *-e/o», Emerita 73 (2): 233-239.

Solinas, P. (2005), «Sul celtico d'Italia: le forme in -u del leponzio», Atti dell'Istituto Veneto di Scienze, Lettere ed Arti 163 (2004-2005). Classe di scienze morali, lettere ed arti: 559-601. Tibiletti Bruno, M.G. (1981), «Le iscrizioni celtiche d'Italia» en E. Campanile (ed.), I Celti d'Italia, Pisa, Giardini: 157-207.

UNTERMANN, J. (2000), «La terminación del genitivo singular de los temas en -o en el celtibérico: de 1965 a 1995», Estudios de lenguas y epigrafia antiguas 3: 125-142.

VAllejo Ruiz, J.M. (2004), «La flexión indoeuropea en -(o)n; algunos datos onomásticos galos e hispanos», Aquitania 20: 133-148.

Villar, F. (ed.; 1990), Studia Indogermanica et Palaeohispanica in honorem A. Tovar et L. Michelena, Salamanca: Universidad, Acta Salmanticensia, Estudios filológicos n. ${ }^{\mathrm{o}} 236$.

- (1995), A New Interpretation of Celtiberian Grammar. Innsbruck: IBS VoKS n. ${ }^{\circ} 62$.

- (1997), «The Celtiberian Language», Zeitschrift für celtische Philologie 49-50: 898-949.

WAtKins, C. $(1998 / 99=2008)$, «A Celtic Miscellany», reimprimido en id., Selected Writings, ed. por L. Oliver, vol. 3, Innsbruck, IBS n. ${ }^{\circ}$ 129: 893-915.

- $(2001=2008)$, «À la suite des perspectives tracées par Michel Lejeune: Aspects du grec et du celtique», reimprimido en id., Selected Writings, ed. por L. Oliver, vol. 3, Innsbruck, IBS n. ${ }^{\circ}$ 129: 990-1000.

Wedenig, R. \& P. de Bernardo Stempel (2005/07), «Keltisches in norischen Personennamen: Namengraffiti aus der Provinz Noricum» en BirKHAN (ed.; 2007): 619-630.

Wodtкo, D.S. (2000), Wörterbuch der keltiberischen Inschriften. Wiesbaden: Reichert, Monumenta linguarum Hispanicarum V/1. 